\title{
Universiteit
}

Leiden

The Netherlands

\section{Impurity states and interlayer tunneling in high temperature superconductors}

Martin, I.; Balatsky, A.V.; Zaanen, J.

\section{Citation}

Martin, I., Balatsky, A. V., \& Zaanen, J. (2002). Impurity states and interlayer tunneling in high temperature superconductors. Physical Review Letters, 88(9), 097003.

doi:10.1103/PhysRevLett.88.097003

Version: $\quad$ Not Applicable (or Unknown)

License: $\quad$ Leiden University Non-exclusive license

Downloaded from: https://hdl.handle.net/1887/65474

Note: To cite this publication please use the final published version (if applicable). 


\title{
Impurity States and Interlayer Tunneling in High Temperature Superconductors
}

\author{
I. Martin, ${ }^{1}$ A. V. Balatsky, ${ }^{1}$ and J. Zaanen ${ }^{2}$ \\ ${ }^{1}$ Theoretical Division, Los Alamos National Laboratory, Los Alamos, New Mexico 87545 \\ ${ }^{2}$ Leiden Institute of Physics, Leiden University, 2333CA Leiden, The Netherlands
}

(Received 22 December 2000; published 15 February 2002)

\begin{abstract}
We argue that the scanning tunneling microscope (STM) images of resonant states generated by doping $\mathrm{Zn}$ or $\mathrm{Ni}$ impurities into $\mathrm{Cu}-\mathrm{O}$ planes of $\mathrm{BSCCO}$ are the result of quantum interference of the impurity signal coming from several distinct paths. The impurity image seen on the surface is greatly affected by interlayer tunneling matrix elements. We find that the optimal tunneling path between the STM tip and the metal $(\mathrm{Cu}, \mathrm{Zn}$, or $\mathrm{Ni}) d_{x^{2}-y^{2}}$ orbitals in the $\mathrm{Cu}-\mathrm{O}$ plane involves intermediate excited states. This tunneling path leads to the fourfold nonlocal filter of the impurity state in $\mathrm{Cu}-\mathrm{O}$ plane that explains the experimental impurity spectra. Applications of the tunneling filter to the $\mathrm{Cu}$ vacancy defects and "direct" tunneling into $\mathrm{Cu}-\mathrm{O}$ planes are also discussed.
\end{abstract}

DOI: $10.1103 /$ PhysRevLett.88.097003

Recently J. C. Davis and collaborators applied the STM technique to image single $\mathrm{Zn}$ and $\mathrm{Ni}$ impurities in optimally doped BSCCO $[1,2]$. These experiments proved that one can image single impurity states in an unconventional superconductor and demonstrated the highly anisotropic structure of these states.

Although it appears that on a gross scale these findings can be understood in terms of a conventional $d$-wave superconductor perturbed by potential scattering, upon closer inspection problems of principle seem to arise. The impurity states observed by STM are characterized by two main features: (1) energy and width of the impurity-induced resonance in the density of states (DOS), and (2) the spatial structure of the resonance. While the DOS seems to be satisfactorily described by a single-site impurity model [3], the real space distribution of intensity cannot be fit by this model. The main problem with the $\mathrm{Zn}$ impurity image seen in the STM experiments is that the intensity of the signal on the impurity site is very bright, which is at odds with the unitary scattering off $\mathrm{Zn}$. We remind the reader that $\mathrm{Zn}^{2+}$ has a closed $d$ shell and hence, exactly on the impurity site the scattering potential is very strong. Unitary scattering is equivalent to the hard wall condition for the conduction states and therefore no or very little intensity of electron states is expected on the $\mathrm{Zn}$ site. A similar problem arises also with explaining the $\mathrm{Ni}$-induced resonance.

Here we demonstrate that these problems find a natural resolution in terms of the specific way in which the local density of states of the cuprate planes is probed in the STM experiments. We argue that the quantum-mechanical nature of the tunneling from the STM tip into the $\mathrm{Cu}-\mathrm{O}$ layer that hosts impurity requires tunneling through the uppermost insulating Bi-O layer which effectively filters the signal. Surprisingly, similar filtering should also take place even in the case of "direct" tunneling into $\mathrm{Cu}-\mathrm{O}$ plane. Such nonlocal tunneling has profound consequences for the real space image of the impurity state seen by STM.

There are two major types of tunneling routes between the STM tip and the conducting orbitals in the $\mathrm{Cu}-\mathrm{O}$ plane:
PACS numbers: $74.25 . \mathrm{Jb}$

(a) direct tunneling due to the overlap between the tip and the planar $3 d_{x^{2}-y^{2}}$ wave functions, and (b) indirect tunneling through intermediate excited (occupied or empty) states, Fig. 1a. The direct tunneling probability over the experimentally relevant distances of about $10 \AA[1,2]$ is, however, exponentially small [4]. On the other hand, the importance of the excited states for mediating STM tunneling follows directly from the $\mathrm{Bi}-\mathrm{O}$ topographs that clearly show the positions of the nominally insulating $\mathrm{Bi}$ atoms on the surface of BSCCO. The analysis of the topographs implies that the excited $\mathrm{Bi}$ orbitals focus the flow of the tunneling electrons. Therefore, we argue that the indirect tunneling via overlapping intermediate orbitals is the dominant tunneling mechanism.

The strongest indirect tunneling channel involves intermediate states that have the largest overlaps. In the case of BSCCO, these are the orbitals that extend out of the planes, such as $4 s$ or $3 d_{3 z^{2}-r^{2}}$ of $\mathrm{Cu}$ and $6 p_{z}$ of Bi. Furthermore, only the states with zero in-plane orbital momentum have nonzero overlap with the apical oxygen $2 p_{z}$ and $3 s$ orbitals that play an important role in the interlayer communication. Being radially symmetric in the $\mathrm{Cu}-\mathrm{O}$ plane, such states cannot couple to the relevant $3 d_{x^{2}-y^{2}}$ states on site. They do couple, however, to the neighboring $3 d_{x^{2}-y^{2}}$ orbitals through the $d$-wave-like fork (Fig. 1b). The resulting tunneling amplitude is

$$
M_{i, j} \sim \Psi_{i+1, j}+\Psi_{i-1, j}-\Psi_{i, j+1}-\Psi_{i, j-1},
$$

where $\Psi_{i, j}$ is the impurity state wave function on site $(i, j)$. Hence, the symmetry analysis leads to the surprising conclusion: Tunneling on top of a particular $\mathrm{Cu}$ (or $\mathrm{Zn}$ or $\mathrm{Ni})$ atom in the $\mathrm{Cu}-\mathrm{O}$ plane does not probe its $3 d_{x^{2}-y^{2}}$ orbitals, but rather measures a linear combination of its neighbors. The tunneling matrix element determines the intensity of the impurity signal $F\left[\Psi_{i, j}\right]=\left|M_{i, j}\right|^{2}$. Calculating the spectral intensities in the vicinity of the impurity using this filtering function reproduces the features seen in the experiments in great detail (Figs. 3 and 4). The 


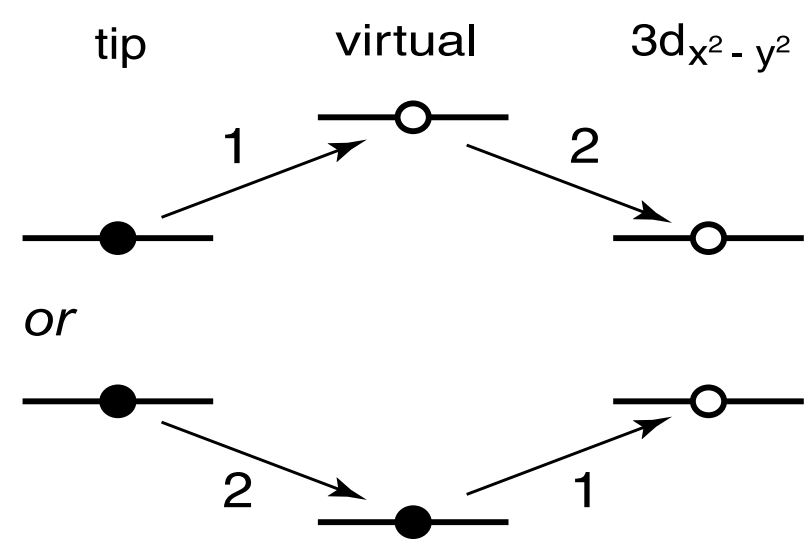

(a)

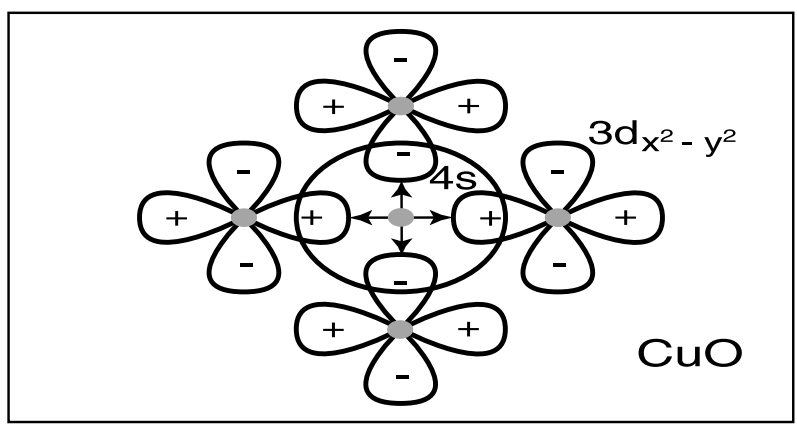

(b)

FIG. 1. (a) Tunneling from STM tip into $3 d_{x^{2}-y^{2}}$ orbitals in the $\mathrm{Cu}-\mathrm{O}$ layer occurs through the virtual energy states of $\mathrm{Bi}$ and $\mathrm{Cu}$. The tunneling processes involve both empty (top) and occupied (bottom) intermediate states. All such processes contribute coherently to the tunneling amplitude from the tip to the $3 d_{x^{2}-y^{2}}$ orbitals. (b) Tunneling through a particular Bi atom does not probe the $3 d_{x^{2}-y^{2}}$ orbital of the metal atom $(\mathrm{Cu}, \mathrm{Zn}$, or $\mathrm{Ni}$ ) right underneath it due to the vanishing overlap. Instead, the tunneling involves orbitals that extend out of the planes, such as $4 s$ (shown) or $3 d_{3 z^{2}-r^{2}}$ of $\mathrm{Cu}$ and $6 p_{z}$ of $\mathrm{Bi}$. These orbitals are symmetric in the $\mathrm{Cu}-\mathrm{O}$ plane and hence couple to the neighboring metal $3 d_{x^{2}-y^{2}}$ orbitals through the $d$-wave-like fork, Eq. (1).

aforementioned anomalies find an explanation in terms of interferences associated with the nonlocal way in which the electronic states are probed. The described filtering mechanism is related to the one responsible for the interlayer tunneling in the bulk cuprates [5,6]. An alternative nonlocal filter based on the incoherent direct tunneling from the tip into $\mathrm{Cu}-\mathrm{O}$ plane was recently studied by Zhu et al. [7]. The angular effect of the filter on the far asymptotic of the impurity states was discussed earlier [8].

Similar to the $\mathrm{Zn}$ case, the Ni impurity tunneling intensity is maximal at the $\mathrm{Bi}$ position immediately above the impurity site. However, there are several important differences between the observed $\mathrm{Zn}$ - and $\mathrm{Ni}$-induced states: (1) The energy of the $\mathrm{Zn}$ state is close to the chemical potential, $\varepsilon_{\mathrm{Zn}}=-2 \mathrm{mV}$, while the $\mathrm{Ni}$ state energy is larger and is split, $\varepsilon_{\mathrm{Ni}}=9$ and $18 \mathrm{mV}$, (2) the $\mathrm{Zn}$ state appears only on the negative bias, while the Ni state shows up both on positive bias and the symmetric negative bias. In this Letter we demonstrate that these experimental features can also be reproduced within the standard theory of the impurity states [3], with the spatial structure of the states being reproduced by properly taking into account the fork effect.

The starting point of our model is a two-dimensional mean-field (MF) Hamiltonian with the nearest neighbor attraction, $V$, which yields $d$-wave superconductivity in the range of dopings close to half filling,

$$
H_{0}=-\sum_{i, j, \sigma} t_{i j} c_{i \sigma}^{\dagger} c_{j \sigma}+\sum_{\langle i j\rangle} c_{i \downarrow} c_{j \dagger} \Delta_{i j}^{*}+\text { H.c. }
$$

Here, $\Delta_{i j}=V\left\langle c_{i \downarrow} c_{j \uparrow}\right\rangle$ is the self-consistent MF superconducting order parameter. The hopping $t_{i j}$ equals $t$ for nearest neighbors and $t^{\prime}$ for the second-nearest neighbor sites $i$ and $j$. The parameters that are relevant for BSCCO are $t=400 \mathrm{meV}, t^{\prime}=-0.3 t$. To match the amplitude of the superconducting gap in optimally doped BSCCO, which is about $40 \mathrm{meV}$, we choose the attraction $V=-0.525 t$. The chemical potential is chosen to yield $16 \%$ doping $(\mu=-t)$.

The local impurity is introduced into Hamiltonian Eq. (2) by modifying the electron energy on a particular site $[3,9-11]$. The corresponding addition to the Hamiltonian is

$$
H_{\text {imp }}=V_{\text {imp }}\left(n_{0 \uparrow}+n_{0 \downarrow}\right)+S_{\text {imp }}\left(n_{0 \uparrow}-n_{0 \downarrow}\right) .
$$

The first term is the potential part of the impurity energy that couples to the total electronic density on site 0 , and the second term describes the magnetic interaction of the impurity spin and the electronic spin density on the same site. We assume that the impurity spin is large and can be treated classically, as if it were a local magnetic field. The goal is to determine $V_{\mathrm{imp}}$ and $S_{\mathrm{imp}}$ so as to match both the location of the impurity states within the gap and the spatial distribution of their intensity.

First let us analyze the position of the impurity energy level as a function of the impurity potential. We solve the MF equations self-consistently on a square lattice with periodic boundary conditions. The results are presented in Fig. 2. All impurities placed in a superconductor generate quasiparticle weight both on positive and symmetric negative biases. The sign of the energy level is defined based on where the majority of the quasiparticle weight resides. Attractive impurities produce energy levels lying below the Fermi surface, while repulsive ones generate states with positive energy. In the limit of a very strong impurity potential both attractive and repulsive impurities generate identical states with a small residual energy related to the amount of the particle-hole symmetry breaking, caused by the specifics of the band structure and doping [12].

The analysis of the energy level positions implies that the $\mathrm{Zn}$ impurity can be associated with a strong attractive potential, $V_{\mathrm{Zn}}=-11 t=-4.4 \mathrm{eV}$ and $\varepsilon_{\mathrm{Zn}}=$ $-0.005 t=-2 \mathrm{meV}$. Ignoring for now the level splitting, the $\mathrm{Ni}$ case can be associated with a relatively weak 


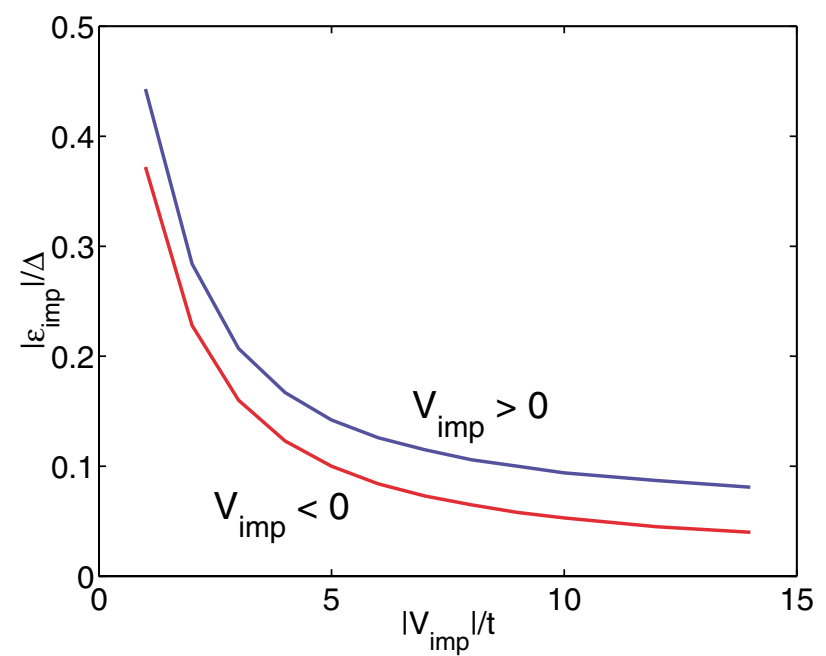

FIG. 2 (color). Impurity energy level position as a function of the potential impurity strength, $V_{\text {imp }}$. The blue (top) line corresponds to repulsive impurities, which produce positive energy levels $\left(\varepsilon_{\text {imp }}>0\right)$; the red line is for attractive impurities, which generate negative energy states $\left(\varepsilon_{\mathrm{imp}}<0\right)$. For strong impurity potential both energies converge to the same small value determined by the amount of particle-hole symmetry breaking.

repulsive impurity, $\quad V_{\mathrm{Ni}}=t$ and $\varepsilon_{\mathrm{Ni}}=0.0443 t=$ $18 \mathrm{meV}$. These impurity strengths are in agreement with the general band structure arguments. $\mathrm{Zn}^{2+}$ ion has 10 electrons that completely fill $d$ orbitals. Hence, the $d_{x^{2}-y^{2}}$ orbital of $\mathrm{Zn}$, relevant for interaction with $\mathrm{Cu}-\mathrm{O}$ plane orbitals, is deep below the Fermi surface. On the other hand, $\mathrm{Ni}^{2+}$ has 8 electrons in the $d$ shell, with the $d_{x^{2}-y^{2}}$ being unoccupied, but with a small energy, given by the level splitting within the $d$ shell.

The spatial distribution of the spectral intensity corresponding to the $\mathrm{Zn}$ impurity is shown in Fig. 3. The top two plots show the intensity $A_{i, j}^{\mathrm{CuO}}$ as it would be seen if STM tip were directly imaging DOS in the $\mathrm{Cu}-\mathrm{O}$ layer. The intensity is related to impurity state wave function $\Psi$ on site $(i, j)$,

$$
A_{i, j}^{\mathrm{CuO}}=\left|\Psi_{i, j}\right|^{2} .
$$

The intensity on the impurity site is suppressed due to the strong impurity potential. The bottom two plots correspond to imaging through the top Bi-O layer. They are obtained by applying a filtering function $F[\Psi]=\left|M_{i, j}\right|^{2}$ to the impurity state wave function $\Psi$. The effect of the filtering function is to produce the intensity

$$
A_{i, j}^{\mathrm{BiO}} \propto\left|\Psi_{i+1, j}+\Psi_{i-1, j}-\Psi_{i, j+1}-\Psi_{i, j-1}\right|^{2} .
$$

Indeed, the intensity on the $\mathrm{Bi}-\mathrm{O}$ layer is maximized on the impurity site due to the interference of the contributions from the impurity's nearest neighbor sites.

The structure of the Ni-induced state is more complicated than the $\mathrm{Zn}$ case. It appears on both positive and negative biases. In addition, there is a peak splitting on each bias. Unlike $\mathrm{Zn}^{2+}, \mathrm{Ni}^{2+}$ impurity is magnetic, with spin 1 . To simulate the effect of spin we include a
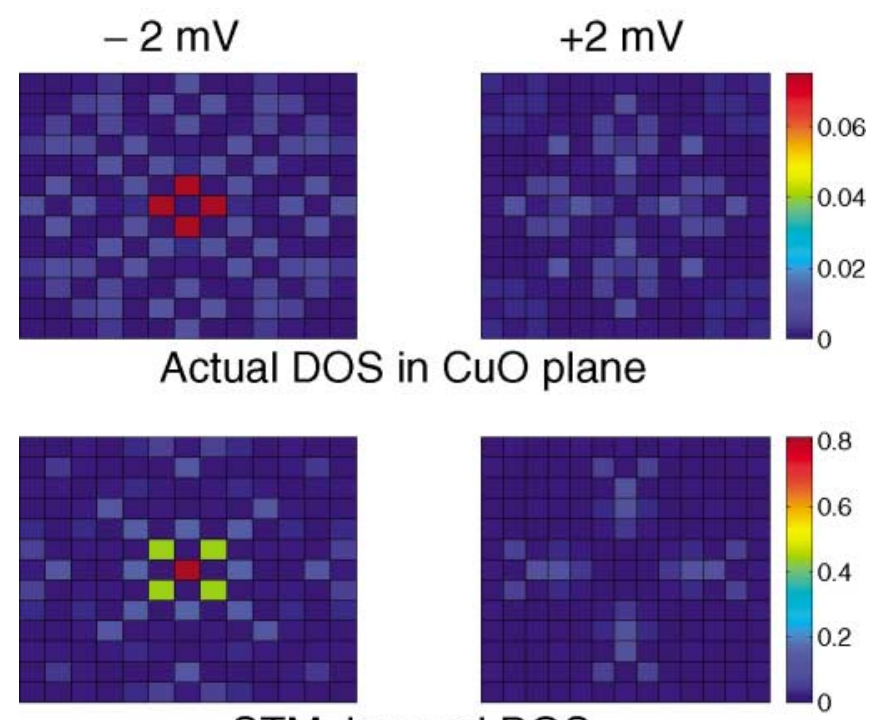

STM-imaged DOS

FIG. 3 (color). Top two plots show the real-space spectral intensity of the calculated $\mathrm{Zn}$ impurity state in the $\mathrm{Cu}-\mathrm{O}$ plane. While most of the intensity is concentrated at the negative bias, $-2 \mathrm{meV}$, as it is seen in the experiments [1], the spatial shape of the state does not agree with the experimental results. The bottom two plots show the same impurity state, but as seen through the Bi-O layer. The figure is obtained by applying the filter function of Eq. (1). Both the energy of the state and the spatial intensity distribution agree with experiment [1].

nonzero magnetic part of the impurity potential, $S_{\mathrm{imp}} \neq 0$, in the Hamiltonian Eq. (3). The spin component introduces level splitting between "up" and "down" spin states. Figure $4 \mathrm{a}$ shows the amplitudes of the spin-split states on the impurity sites and its neighbors for $V_{\mathrm{Ni}}=t$ and $S_{\mathrm{Ni}}=0.4 t$. The spin-split energy levels are $\varepsilon_{\downarrow}=0.052 t$ and $\varepsilon_{\uparrow}=0.037 t$. The total spin-up and spin-down intensities for each bias are shown in Fig. 4b. Both Figs. 4a and $4 \mathrm{~b}$ correspond to the image affected by the fork filter. The general shape of the states agrees well with the experimental data [2]. The total weight on the negative bias is about a factor of 3 smaller than the weight on the positive bias.

Some aspects of the Ni-impurity state cannot be addressed within the simple framework of our theory. We assumed that the spin of the impurity is static and plays the role of a local magnetic field. Bulk measurements indicate that $\mathrm{Ni}$ dopants do not introduce additional magnetic moments. This may be either due to the partial Kondo screening of $S=1 \mathrm{Ni}$ spin or due to the local attraction of additional hole ("self-doping") by $\mathrm{Ni}^{2+}$ to form an effective $\mathrm{Ni}^{3+}$ complex with spin $1 / 2$. No magnetic signature, combined with the weak potential character of $\mathrm{Ni}$, may explain the relatively weak pair-braking effect of $\mathrm{Ni}$ doping observed experimentally.

In the case of $\mathrm{Zn}$, we focused on the large local potential scattering off the impurity as a main effect to account for the STM intensity distribution. It is important to mention that $\mathrm{Zn}$ substitution also induces local magnetic moment, 
(a)
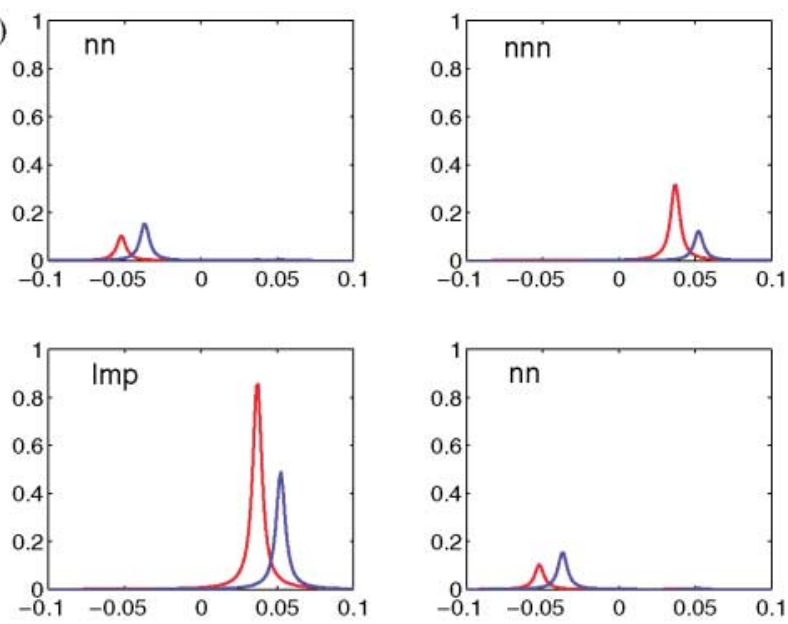

(b)
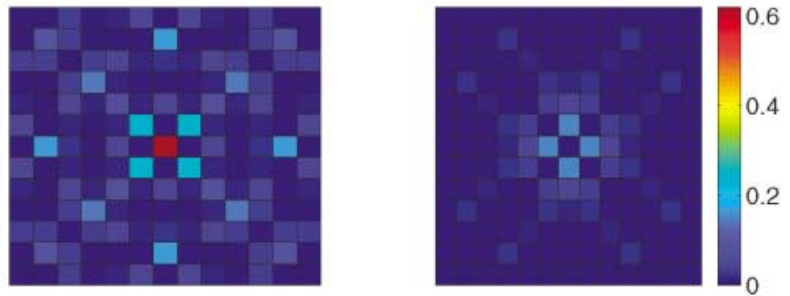

FIG. 4 (color). Simulation of $\mathrm{Ni}$ as an impurity with weak mixed potential, $V_{\mathrm{Ni}}=t$ and $S_{\mathrm{Ni}}=0.4 t$. (a) Spectral intensity on the impurity site, its nearest neighbors (nn), and next nearest neighbor (nnn). The red line is spin up, and the blue line is spin down. (b) Intensity map for the combined spin up and spin down states.

which is observed in magnetic susceptibility and NMR measurements [13]. The quenching of magnetic moment around $\mathrm{Zn}$ can lead to the Kondo resonance near the Fermi surface, as has been proposed by Polkovnikov et al. [14]. Below the Kondo temperature, $\mathrm{Zn}$ should behave as a unitary scatterer, virtually indistinguishable from either local or extended $[14,15]$ potential scatterer. We argue, however, that the tunneling fork filter should apply regardless of the details of the scattering mechanism.

The tunneling fork mechanism has consequences beyond the settings of the original experiments [1,2]. Here we point out two of them: (1) the direct tunneling into $\mathrm{Cu}-\mathrm{O}$ plane, and (2) the structure of the $\mathrm{Cu}$ vacancy states. One could assume that the tunneling into the exposed top $\mathrm{Cu}-\mathrm{O}$ plane should be free of the tunneling fork. However, upon closer inspection it is clear that even in this case, the direct tunneling from tip into the planar $3 d_{x^{2}-y^{2}}$ orbitals is exponentially weak compared to the indirect tunneling through the $s$-wave-like orbitals of $\mathrm{Cu}, \mathrm{Zn}$, or $\mathrm{Ni}$ extending out of the plane. Hence, the tunneling fork should apply also in this case, resulting in the same spatial form of the filtered impurity states as obtained above. In the case of
$\mathrm{Cu}$ vacancy in the $\mathrm{Cu}-\mathrm{O}$ plane, under the assumption that there are no $s$-wave-like orbitals centered at the vacancy site, the tunneling fork mechanism implies that the resonance state observed by STM should be the same as for Zn, but with no spectral intensity in the center of the pattern.

In conclusion, we have demonstrated that recently observed $\mathrm{Zn}$ and Ni impurity states in BSCCO [1,2] can be explained by a simple model of strong potential impurity in the case of $\mathrm{Zn}$ and mixed (potential + spin) impurity in the case of $\mathrm{Ni}$, interacting with a $d$-wave superconducting condensate. The crucial aspect that we have included in the present treatment is the effect of the quantum-mechanical tunneling between the STM tip and the $\mathrm{Cu}-\mathrm{O}$ planes. The same nonlocal filter effect should be operable in the case of the "direct" tunneling into $\mathrm{Cu}-\mathrm{O}$ planes and should have consequences for other types of defects, e.g., Cu vacancies.

We are grateful to J.C. Davis, S. H. Pan, S. Sachdev, M. Vojta, and J.X. Zhu for useful discussions. This work has been supported by the U.S. DoE.

[1] S. H. Pan, E. W. Hudson, K. M. Lang, H. Eisaki, S. Uchida, and J. C. Davis, Nature (London) 403, 746 (2000).

[2] E. W. Hudson, K. M. Lang, V. Madhavan, S. H. Pan, H. Eisaki, S. Uchida, and J.C. Davis, Nature (London) 411, 920 (2001).

[3] A. V. Balatsky, M. I. Salkola, and A. Rosengren, Phys. Rev. B 51, 15547 (1995); A. V. Balatsky and M. I. Salkola, Phys. Rev. Lett. 76, 2386 (1996); M. Salkola, A. V. Balatsky, and J. R. Schrieffer, Phys. Rev. B 55, $12648-12661$ (1997).

[4] J. Tersoff and D. R. Hammann, Phys. Rev. B 31, 805 (1985).

[5] S. Chakravarty, A. Sudbo, P. W. Anderson, and S. Strong, Science 261, 337 (1993).

[6] O. K. Andersen, A. I. Lichtenstein, O. Jepsen, and F. Paulsen, J. Phys. Chem. Solids 56, 1573 (1995).

[7] J. X. Zhu, C. S. Ting, and C. R. Hu, Phys. Rev. B 62, 6027 (2000).

[8] A. V. Balatsky, Nature (London) 403, 717 (2000).

[9] L. Yu, Acta Phys. Sin. 21, 75 (1965); H. Shiba, Prog. Theor. Phys. 40, 435 (1968).

[10] A. Yazdani, B. A. Jones, C. P. Lutz, M. F. Crommie, and D. M. Eigler, Science 275, 1767 (1997).

[11] J. M. Byers, M. E. Flatte, and D. J. Scalapino, Phys. Rev. Lett. 71, 3363 (1993).

[12] H. V. Kruis, I. Martin, and A. V. Balatsky, Phys. Rev. B 64, 054501 (2001).

[13] H. Alloul et al., Phys. Rev. Lett. 67, 3140 (1991); A. V. Mahajan et al., Europhys. Lett. 46, 678 (2000); M-H. Julien et al., Phys. Rev. Lett. 84, 3422 (2000).

[14] A. Polkovnikov, S. Sachdev, and M. Voita, Phys. Rev. Lett. 86, 296 (2001).

[15] J. X. Zhu and C. S. Ting, Phys. Rev. B 63, 020506 (2001). 